\title{
Leptophilic neutral Higgs bosons in two Higgs doublet model at a linear collider
}

\author{
Majid Hashemi ${ }^{\mathrm{a}}$ \\ Physics Department, College of Sciences, Shiraz University, Shiraz 71946-84795, Iran
}

Received: 14 January 2017 / Accepted: 26 April 2017 / Published online: 11 May 2017

(C) The Author(s) 2017. This article is an open access publication

\begin{abstract}
This paper addresses the question of the observability of neutral Higgs bosons through the leptonic decay in a two Higgs doublet model (2HDM). Both scalar and pseudoscalar Higgs bosons $(H, A)$ are considered. The model is set to type IV to enhance the leptonic decay. In such a scenario, a signal production process like $e^{+} e^{-} \rightarrow A^{0} H^{0} \rightarrow \tau \tau \mu \mu$ or $\mu \mu \tau \tau$ would provide a clear signal on top of the background in a di-muon invariant mass distribution far from the $Z$ boson pole mass. The analysis is based on a $\tau$-id algorithm which preselects events if they have two $\tau$ jets by requiring a hadronic $\tau$ decay. Several benchmark points are defined for the search, requiring a linear collider operating at $\sqrt{s}=0.5$ and $1 \mathrm{TeV}$. It is shown that the signal can be observed on top of the background in all benchmark points at an integrated luminosity of $1000 \mathrm{fb}^{-1}$.
\end{abstract}

\section{Introduction}

The standard model (SM) of particle physics has been tested successfully in a large number of experiments. The Higgs boson introduced through a single $\mathrm{SU}(2)$ doublet [1-6] has been observed at LHC $[7,8]$ with a mass near $125 \mathrm{GeV}$. The ongoing analyses at LHC confirm that the observed boson has the same properties as expected for a Standard Model Higgs boson. However, it is still an open question whether the observed particle belongs to a single $\mathrm{SU}(2)$ doublet or a richer framework such as a two Higgs doublet model [9-11].

The two Higgs doublet models are well motivated beyond SM in different aspects. They provide the basis for building weakly coupled theories such as the minimal supersymmetric extension of standard model (MSSM) [12-14] as well as the strongly coupled composite Higgs model [15]. There are four types of 2HDMs, which are designed to accommodate different possible scenarios of Higgs-fermion couplings. The

a e-mail: majid.hashemi@cern.ch ratio of vacuum expectation values of the two Higgs doublets $\left(\tan \beta=v_{2} / v_{1}\right)$ is a key parameter in all 2HDM types [16].

In general, 2HDMs (and MSSM) involve more than a single physical Higgs boson due to the second Higgs doublet, which is added to the SM resulting in more degrees of freedom available to the model after giving masses to the gauge bosons. Taking the lightest scalar, $h$, as the SM-like Higgs boson, there are two more neutral Higgs bosons, $H, A$, and two charged bosons, $H^{ \pm}$. Contrary to the MSSM which involves almost degenerate heavy Higgs boson states, the 2HDM allows for different Higgs boson masses, thus providing a broader parameter space available for study. The theory and phenomenology of 2HDMs has been discussed in detail in [17]. The 2HDM is also useful in flavor physics for bringing theoretical predictions close to experimental observations by including processes which involve 2HDM Higgs bosons [18].

In this work, different benchmark points are defined in the Higgs boson mass spectrum space and a search is performed at a linear collider. The signal process is taken as a joint $A H$ production through $e^{+} e^{-} \rightarrow A H \rightarrow \mu \mu \tau \tau$ or $\tau \tau \mu \mu$. It is expected that a leptonic decay of the neutral Higgs $(H$ or $A$ ) would provide a clear signal due to the reasonable lepton reconstruction efficiency at linear colliders. For such a process, the $2 \mathrm{HDM}$ type IV is chosen to enhance the leptonic Higgs decay at high $\tan \beta$. There has recently been work searching for the same signal at LHC [19]. In this analysis, it is shown that signals of both $H$ and $A$ bosons are observable at a future linear collider. Details of the model and benchmark points are presented in the next section.

\section{The Higgs sector of $2 \mathrm{HDM}$}

The Higgs sector of 2HDM involves neutral and charged Higgs couplings with fermions (leptons and quarks). The 
Lagrangian for the neutral Higgs-fermion couplings as introduced in [20] is written

$$
\begin{aligned}
-\mathcal{L}= & \frac{1}{\sqrt{2}} \bar{D}\left\{\kappa^{D} s_{\beta-\alpha}+\rho^{D} c_{\beta-\alpha}\right\} D h \\
& +\frac{1}{\sqrt{2}} \bar{U}\left\{\kappa^{U} s_{\beta-\alpha}+\rho^{U} c_{\beta-\alpha}\right\} U h \\
& +\frac{1}{\sqrt{2}} \bar{L}\left\{\kappa^{L} s_{\beta-\alpha}+\rho^{L} c_{\beta-\alpha}\right\} L h \\
& +\frac{1}{\sqrt{2}} \bar{D}\left\{\kappa^{D} c_{\beta-\alpha}-\rho^{D} s_{\beta-\alpha}\right\} D H \\
& +\frac{1}{\sqrt{2}} \bar{U}\left\{\kappa^{U} c_{\beta-\alpha}-\rho^{U} s_{\beta-\alpha}\right\} U H \\
& +\frac{1}{\sqrt{2}} \bar{L}\left\{\kappa^{L} c_{\beta-\alpha}-\rho^{L} s_{\beta-\alpha}\right\} L H \\
& +\frac{i}{\sqrt{2}} \bar{D} \gamma_{5} \rho^{D} D A-\frac{i}{\sqrt{2}} \bar{U} \gamma_{5} \rho^{U} U A \\
& +\frac{i}{\sqrt{2}} \bar{L} \gamma_{5} \rho^{L} L A
\end{aligned}
$$

where $U, D, L$ are up-type quark, down-type quark and lepton fields, $h, H, A$ are neutral Higgs boson fields, $\kappa^{f}=\sqrt{2} \frac{m_{f}}{v}$ for any fermion type $f, \rho^{f}$ are parameters proportional to $\kappa^{f}$ through $\tan \beta$ or $\cot \beta$ factors and $s_{\beta-\alpha}$ and $c_{\beta-\alpha}$ are abbreviations for $\sin (\beta-\alpha)$ and $\cos (\beta-\alpha)$, respectively.

The four types of interactions (2HDM types) depend on the values of $\rho^{f}$ and are defined as in Table 1 [21]. Type III is sometimes called "flipped" or "type Y" and type IV is also known as "lepton-specific" or "type X". The collider phenomenology of 2HDM depends on the model type which determines which kinds of Higgs-fermion interactions are more important for a given $\tan \beta$ [22].

In order to respect SM observations, the lightest Higgs boson, $h$, is taken to be SM-like by setting $s_{\beta-\alpha}=1$. This

\begin{tabular}{|c|c|c|c|c|}
\hline & \multicolumn{4}{|l|}{ Type } \\
\hline & I & II & III & IV \\
\hline$\rho^{D}$ & $\kappa^{D} \cot \beta$ & $-\kappa^{D} \tan \beta$ & $-\kappa^{D} \tan \beta$ & $\kappa^{D} \cot \beta$ \\
\hline$\rho^{U}$ & $\kappa^{U} \cot \beta$ & $\kappa^{U} \cot \beta$ & $\kappa^{U} \cot \beta$ & $\kappa^{U} \cot \beta$ \\
\hline$\rho^{L}$ & $\kappa^{L} \cot \beta$ & $-\kappa^{L} \tan \beta$ & $\kappa^{L} \cot \beta$ & $-\kappa^{L} \tan \beta$ \\
\hline
\end{tabular}
ensures that the $s_{\beta-\alpha}$ factor in the lightest Higgs-gauge coupling is set to unity, while the heavier Higgs boson, $H$, decouples from gauge bosons [17]. On the other hand, terms containing $\rho$ are eliminated to remove the $\tan \beta$ dependence of the SM-like Higgs-fermion interactions. Therefore all SM-

Table 1 Different types of 2HDM in terms of the Higgs boson couplings with $U$ (up-type quarks), $D$ (down-type quarks) and $L$ (leptons) like Higgs interactions with fermions and gauge bosons are equal to their SM corresponding values.

Among the two types I and IV, type I is interesting for low $\tan \beta$ as all couplings in the neutral Higgs sector are proportional to $\cot \beta$ and the leptonic decays are also suppressed. A study of neutral Higgs decays in this type at LHC shows that a pseudo-scalar Higgs production followed by the decay $A \rightarrow Z H$ can be observed with $H$ decaying to $b \bar{b}$ or $W W$ [23].

On the other hand, type IV provides a Higgs-lepton coupling which is enhanced as $\tan \beta$. Therefore at high $\tan \beta$ all neutral Higgs, $H$, couplings are suppressed except for the leptonic decays. Such a "leptophilic" Higgs can be observed in a di-lepton invariant mass distribution on top the background. The lepton in this case is either $\tau$ or $\mu$. The branching ratio of Higgs decay to $\tau$ is higher because of the larger lepton mass. However, identification of such decays requires a hadronic $\tau$-id which reconstructs the hadronic part of the decay. On the other hand, the muonic decay is easy to reconstruct and observe, as the muon trajectory is well identified at a linear collider. The Higgs branching ratio of decay to muons is 3 permil in the best case.

\section{Benchmark points and cross sections}

In order to select working points, it is better to start with plots of branching ratio of $H$ and $A$ decays in a 2HDM type IV as shown in Figs. 1 and 2. The scalar $H$ decays to $\tau$ or $\mu$ for masses below the threshold of top pair production, i.e., $m_{H} \simeq 350 \mathrm{GeV}$. A heavier Higgs boson would prefer to decay to $t \bar{t}$. Therefore the analysis is limited to $m_{H}$ below the top pair threshold. The pseudo-scalar Higgs $A$ also decays to $\tau$ and $\mu$ until $A \rightarrow Z H$ starts to be kinematically possible.

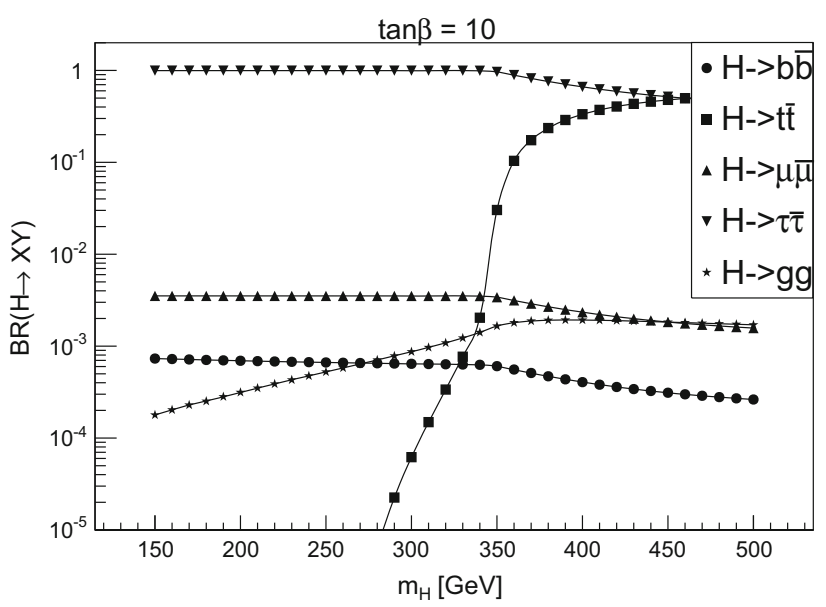

Fig. 1 The $H$ branching ratio of decays as a function of its mass 


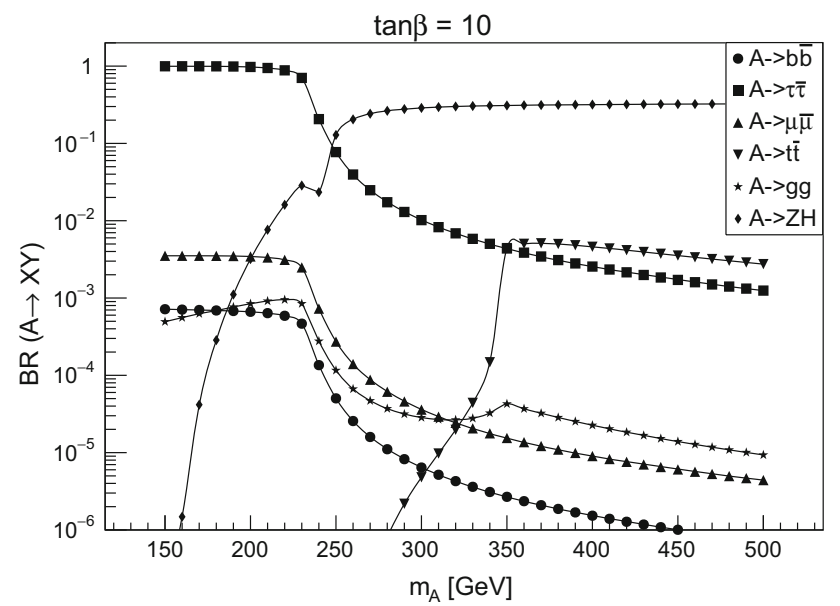

Fig. 2 The $A$ branching ratio of decays as a function of its mass $\left(m_{H}=\right.$ $150 \mathrm{GeV}$ )

Table 2 The benchmark points selected for linear colliders operating at $\sqrt{s}=500$ and $1000 \mathrm{GeV}$. The charged Higgs mass is set to the heaviest boson mass. The BP5 is the same point as BP4 but searched for at a different center of mass energy

\begin{tabular}{|c|c|c|c|c|c|c|}
\hline $\mathrm{BP}$ & $m_{h}$ & $m_{H}$ & $m_{A}$ & $m_{H^{ \pm}}$ & $s_{\beta-\alpha}$ & $\tan \beta$ \\
\hline \multicolumn{7}{|c|}{$\sqrt{s}=500 \mathrm{GeV}$} \\
\hline 1 & 125 & 150 & 150 & 150 & 1 & 10 \\
\hline 2 & & 150 & 200 & 200 & & \\
\hline 3 & & 200 & 200 & 200 & & \\
\hline 4 & & 200 & 250 & 250 & & \\
\hline \multicolumn{7}{|c|}{$\sqrt{s}=1000 \mathrm{GeV}$} \\
\hline 5 & 125 & 200 & 250 & 250 & 1 & 10 \\
\hline 6 & & 250 & 300 & 300 & & \\
\hline 7 & & 300 & 300 & 300 & & \\
\hline 8 & & 300 & 350 & 350 & & \\
\hline
\end{tabular}

The scenario followed in this paper assumes that the pseudo-scalar Higgs, $A$, is heavier than $H$. For a given $m_{H}$, if $m_{A}$ increases to regions where $m_{A}>m_{H}+m_{Z}$, the type independent decay $A \rightarrow Z H$ occurs, resulting in a suppression of leptonic decays. Therefore all benchmark points are chosen not to allow such a decay to overwhelm the leptonic decays. The mass difference $m_{A}-m_{H}$ is thus adjusted to be less than $m_{Z}$, leaving no phase space for the decay products.

A study of LHC discovery potential for a leptophilic Higgs boson shows that neutral Higgs boson masses below $140 \mathrm{GeV}$ is observable at $30 \mathrm{fb}^{-1}$ [24]. In the current work, the attempt is to search for moderate and high masses of neutral Higgs bosons. Therefore the analysis covers linear colliders with $\sqrt{s}=0.5$ and $1 \mathrm{TeV}$. The selected benchmark points (BP's) are presented in Table 2 . They cover $H$ masses in the range $150-300 \mathrm{GeV}$. The charged Higgs mass is set to $m_{A}$ to reduce $\Delta \rho$ [25]. In addition to check the $\Delta \rho$ value, all points are verified to be consistent with the potential stability, perturbativity and unitarity and current experimental limits on Higgs boson masses using 2HDMC 1.6.3 [26,27]. All above conditions are met by the selected benchmark points.

Moreover, all selected points are in agreement with theoretical and experimental constraints. The experimental constraints on the light neutral and charged Higgs bosons come from LEP [28-30] summarized as $m_{H^{ \pm}} \geq 78.6 \mathrm{GeV}$ and $m_{A} \geq 93.4 \mathrm{GeV}$. Since the scope of this analysis is focused on heavier Higgs boson masses $\left(m_{H / A / H^{ \pm}}>150 \mathrm{GeV}\right)$, these experimental limits are already satisfied and impose no problem for the analysis domain. Moreover, these results are based on MSSM (2HDM type II with supersymmetry), which is different from what is considered in this analysis.

The heavy neutral Higgs boson has been searched for at LHC [31,32]. The neutral Higgs boson mass in the range $m_{H / A}=200-400 \mathrm{GeV}$ has been excluded for $\tan \beta \geq 5$. However, this result is again based on MSSM. The MSSM constrains the Higgs boson masses, especially at high values where they are almost degenerate. Therefore the mass spectrum (as well as the Higgs-fermion couplings) are different from what is considered at a general $2 \mathrm{HDM}$ and experimental limits cannot easily be applied to a $2 \mathrm{HDM}$ of a different type.

Incorporating flavor physics data results, types II and III will receive a strong lower limit on the charged Higgs mass at $480 \mathrm{GeV}$ [33]. This is due to the fact that many of the flavor observables (including heavy meson decays) receive deviations from their corresponding SM values by adding diagrams in which the $W^{ \pm}$boson is replaced by a charged Higgs boson, $H^{ \pm}$. The charged Higgs contribution in such processes is enhanced with increasing $\tan \beta$ in type II and III due to $\tan \beta$ dependence of the charged Higgs-quark couplings. The corresponding coupling in types I and IV behaves opposite to that in types II and III and is proportional to $\cot \beta$. Therefore the charged Higgs limits from flavor physics in types I and IV are very soft and basically relevant at $\tan \beta$ values as low as 2 . As a conclusion, the analysis presented in this work is safe from flavor physics limits on $2 \mathrm{HDM}$ charged Higgs boson mass.

The first four points are studied at $\sqrt{s}=0.5 \mathrm{TeV}$ and the rest at $\sqrt{s}=1 \mathrm{TeV}$. The BP4 and BP5 are in fact the same points. As will be shown, this point is not observable at 0.5 $\mathrm{TeV}$, but it has a signal exceeding $5 \sigma$ at $1 \mathrm{TeV}$.

The signal cross sections are shown in Figs. 3 and 4. The signal process, $e^{+} e^{-} \rightarrow A H$, depends on $s_{\beta-\alpha}$, which is set to 1 . Therefore increasing $\tan \beta$ has no effect on the production cross section. On the other hand, the leptonic decays of $A$ and $H$ are dominated by $\tau$ leading to $\operatorname{BR}(H \rightarrow \tau \tau) \simeq 1$ at $\tan \beta \simeq 10$. Again increasing $\tan \beta$ to higher values has no sizable effect on the signal rate through the Higgs boson decays. The signal cross section is sensitive to the mass difference $\delta=m_{A}-m_{H}$ and is reduced when $\delta$ decreases. Therefore a high cross section prefers a large mass split- 


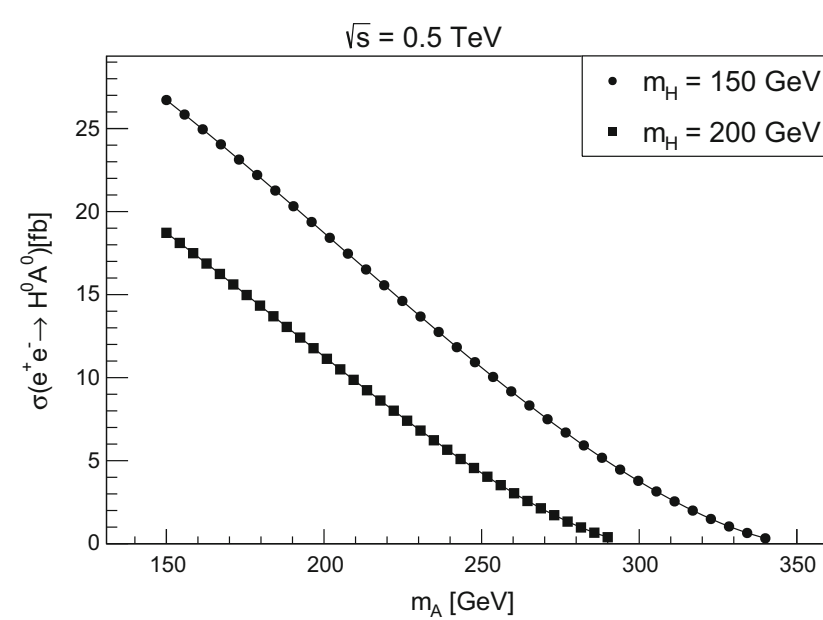

Fig. 3 The signal cross section at $\sqrt{s}=0.5 \mathrm{TeV}$ as a function of the two neutral Higgs boson masses

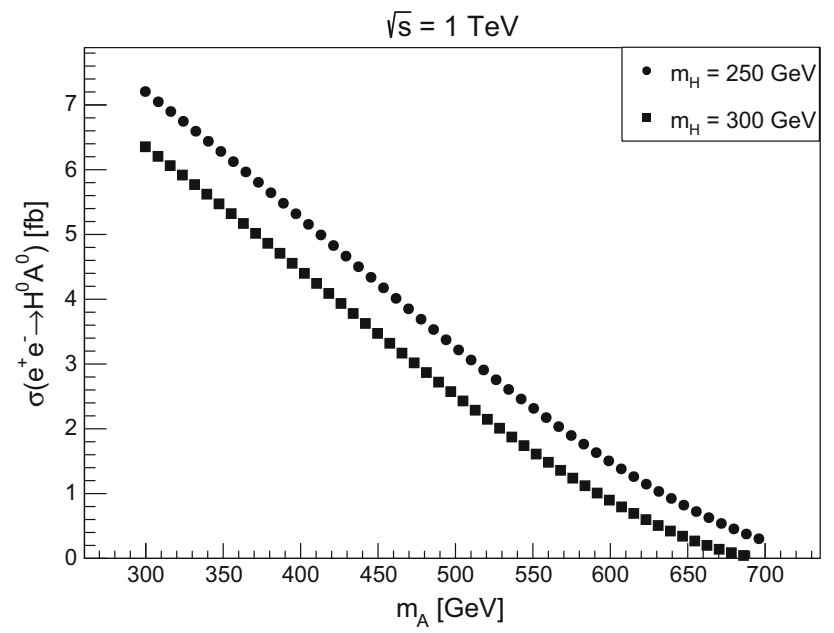

Fig. 4 The signal cross section at $\sqrt{s}=1 \mathrm{TeV}$ as a function of the two neutral Higgs boson masses

ting which is avoided by the requirement of leptonic decay enhancement and $A \rightarrow Z H$ suppression.

The main SM background processes are $W W, Z Z, Z / \gamma^{*}$ and $t \bar{t}$. The signal and background cross sections are all listed in Table 3 at two center of mass energies.

\section{Signal selection and analysis}

Signal and background events are generated using PYTHIA 8 [34]. Jets are reconstructed using FASTJET 2 . $8[35,36]$ based on the anti- $k_{T}$ algorithm with a cone size of 0.4 . The jet energy is smeared according to an energy resolution of $\sigma / E=3.5 \%$ predicted for CLIC [37]. Only jets which pass the following kinematic threshold are selected:

$E_{T}^{\mathrm{jet}}>10 \mathrm{GeV},|\eta|<1.5$.
Table 3 The signal and background cross sections at $\sqrt{s}=0.5$ and $1 \mathrm{TeV}$

\begin{tabular}{lllll}
\hline$\sqrt{s}=0.5 \mathrm{TeV}$ & & & & \\
Signal & $\mathrm{BP} 1$ & $\mathrm{BP} 2$ & $\mathrm{BP} 3$ & $\mathrm{BP} 4$ \\
$\sigma[f b]$ & 26.7 & 18.7 & 11.3 & 4.3 \\
Background & $\mathrm{WW}$ & $\mathrm{ZZ}$ & $Z / \gamma^{*}$ & $t \bar{t}$ \\
$\sigma[p b]$ & 7.83 & 0.58 & 16.67 & 0.59 \\
$\sqrt{s}=1 \mathrm{TeV}$ & & & & \\
Signal & $\mathrm{BP} 1$ & $\mathrm{BP} 2$ & $\mathrm{BP} 3$ & $\mathrm{BP} 4$ \\
$\sigma[f b]$ & 8.8 & 7.19 & 6.35 & 5.42 \\
Background & $\mathrm{WW}$ & $\mathrm{ZZ}$ & $Z / \gamma^{*}$ & $t \bar{t}$ \\
$\sigma[p b]$ & 3.19 & 0.2 & 4.3 & 0.21 \\
\hline
\end{tabular}

The $b$-tagging is applied on jets to veto sources of $b$-jets in background events. The algorithm is based on matching $b$-jets with a generated $b$-quark in the event flying collinearly with a spatial separation $\Delta R<0.2$. The $b$-tagging efficiency is set to $60 \%$ for $b$-quarks and $10 \%$ for $c$-quarks. An event is selected if no $b$-jet is present in it. This requirement is called the $b$-jet veto.

The reconstructed jets make the seed for the $\tau$ identification. The $\tau$ jets are characterized as isolated narrow jets because they consist of few charged particles (pions) flying collinearly. A normal jet from QCD interactions accommodates a large number of tracks. The $\tau$ identification algorithm starts with the isolation requirement which verifies that there is no track with $p_{T}>1 \mathrm{GeV}$ in an annulus defined by $0.07<\Delta R<0.1$ around the jet's hottest track. The next key feature is the number of tracks inside the signal cone defined as a narrow cone $(\Delta R=0.07)$ around the hottest track. Since $\tau$ jets predominantly decay to one or three charged pions in their hadronic decays, there should be 1 or 3 tracks inside the signal cone.

The muon selection is based on finding muons in the central region and above a transverse energy threshold as follows:

$E_{T}^{\text {muon }}>20 \mathrm{GeV},|\eta|<1.5$.

The muon track momentum resolution is also applied as $\sigma_{p_{T}} / p_{T}^{2}=2 \times 10^{-5} \mathrm{GeV}^{-1}[37]$.

The number of each of the physical objects (jet or lepton) in the event has to satisfy the following set of requirements:

Number of jets $=2$ or more

Number of b-jets $=0$

Number of $\tau$-jets $=2$ or more

Number of muons $=2$ or more

The two muons from neutral Higgs bosons are produced back-to-back in the Higgs boson decay frame. Since there 


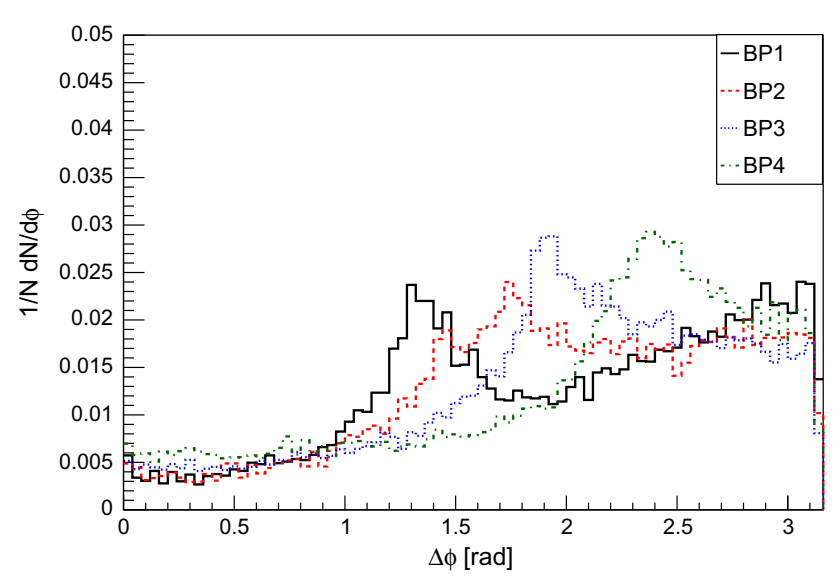

Fig. $5 \Delta \phi$ distribution from the muon pair in signal events from BP1 to $\mathrm{BP} 4$

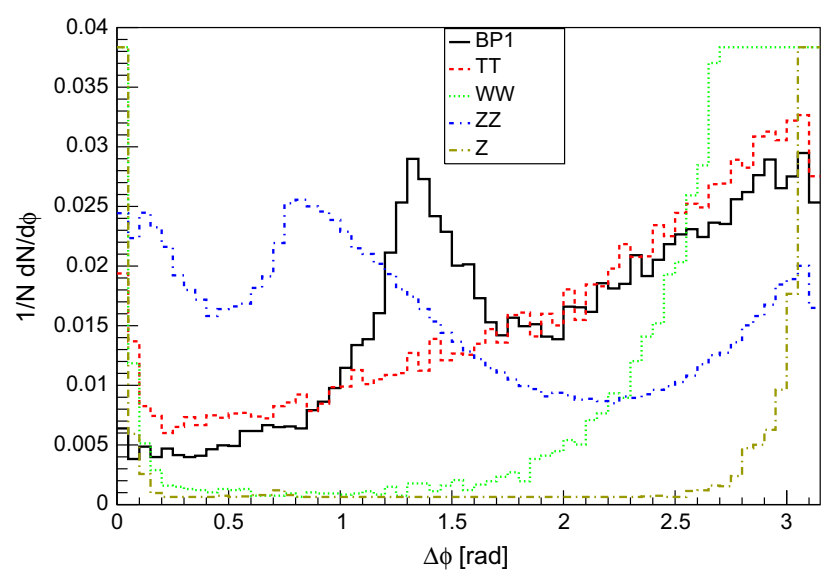

Fig. $6 \Delta \phi$ distribution from the muon pair in signal (BP1) and background events

is a limited center of mass energy, a small kinetic energy is transfered to each neutral Higgs boson in the hard scattering. The available energy becomes smaller if the Higgs boson mass is increased.

The $\Delta \phi$ between the two muons in signal events is shown in Fig. 5 for the first four benchmark points. As seen, the bump (from true muon pairs) shifts to larger values (near $\pi$ ) when moving from BP1 to BP4 and this reflects the fact that the produced Higgs bosons can be considered at rest when the total mass of them tends to the center of mass energy of the system. Figure 6 shows the same distribution for signal and background events at $\sqrt{s}=0.5 \mathrm{TeV}$.

The $Z Z$ background also appears to follow the same effect but in this case the two muons are more collinear (smaller $\Delta \phi$ ) due to the large Lorentz boost the $Z$ boson acquires. There are, however, two peaks in $\Delta \phi$ distribution of $\mathrm{ZZ}$ events. The peak at low values $(\Delta \phi<0.5)$ is related to the off-shell $Z^{*}$ or virtual photons $\left(\gamma^{*}\right)$, which can be verified by plotting the invariant mass of the two muons versus $\Delta \phi$ in a two dimensional plot as shown in Fig. 7. As seen

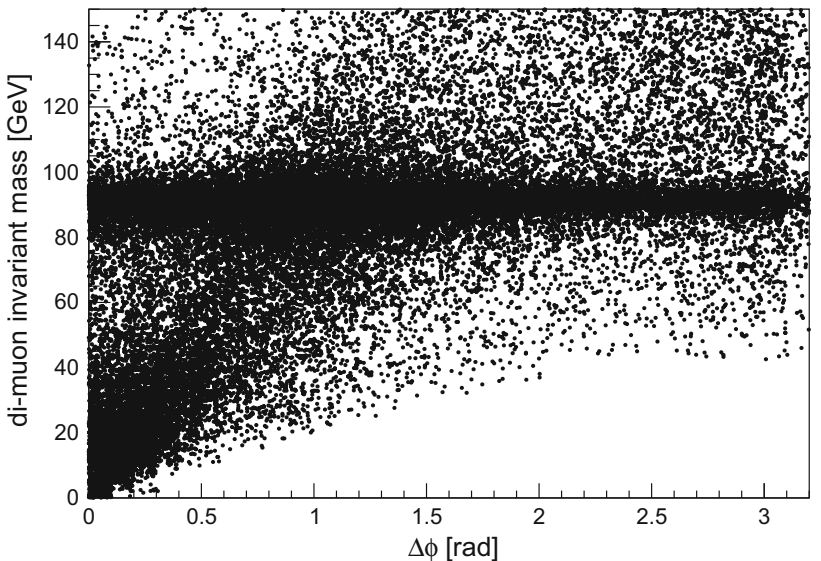

Fig. 7 Invariant mass of the two muons in $\mathrm{ZZ}$ events vs. $\Delta \phi$

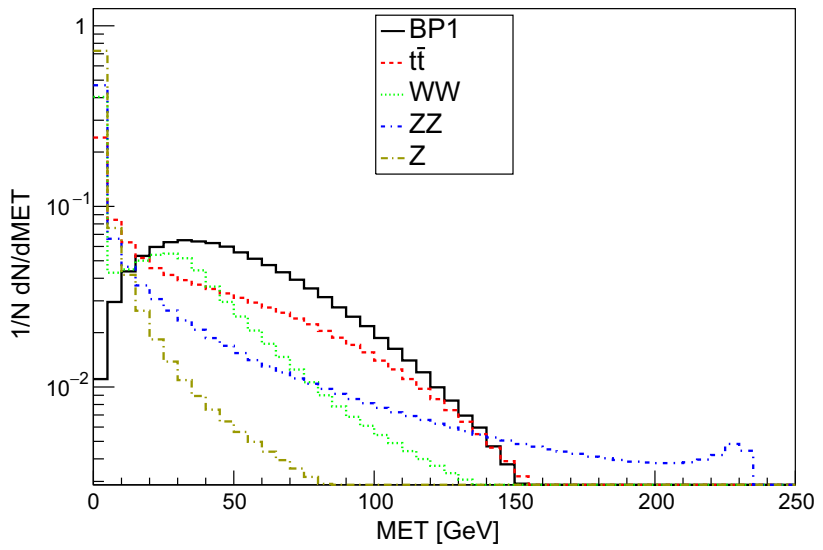

Fig. 8 The missing transverse energy in signal and background events

from Fig. 7 (and verified by generator level information), low $\Delta \phi$ values correspond to the off-shell $Z^{*}$ candidates or photons. The region of interest in the di-muon invariant mass distribution is the region above the resonance $Z$ peak. Therefore, events with small di-muon invariant mass will be finally suppressed in the analysis. Any cut on $\Delta \phi$ at this stage is intended to suppress the $W W$ events as well as single gauge boson events $\left(Z / \gamma^{*}\right)$, while keeping the signal statistics. Based on the above considerations the following selection cut is applied to suppress the background events:

$0.1<\Delta \phi_{\text {(two muons) }}<2.6$.

The missing transverse energy is also calculated for signal and background events as shown in Fig. 8. Based on Fig. 8 a threshold on MET is applied,

MET $>50 \mathrm{GeV}$.

This requirement is due to the fact that $\tau$ jets in signal events produce a source of neutrino (missing transverse energy) when they decay hadronically. However, in processes 


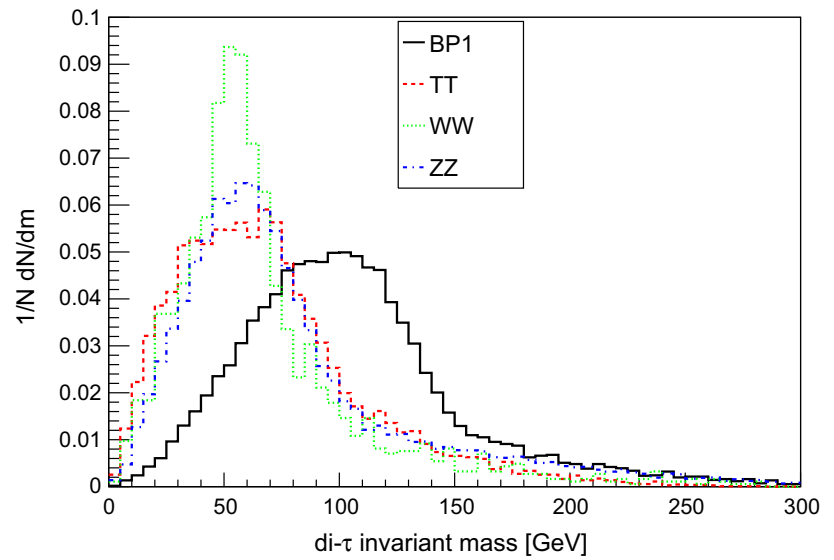

Fig. 9 The di- $\tau$ invariant mass distribution in signal and background events. The $Z / \gamma$ background has been eliminated as there are few events of this type and they are rejected easily by further cuts

like a Drell-Yan background, the missing transverse energy is very small and these background events are dramatically suppressed by this cut.

Since one of the Higgs bosons decays to a pair of $\tau$-jets, the di- $\tau$ invariant mass is higher than the usual corresponding values from $\mathrm{SM}$ processes such as $Z$ or $Z Z$ production, as shown in Fig. 9. Therefore a threshold is applied on the di- $\tau$ invariant mass as follows:

di- $\tau$ invariant mass $>50 \mathrm{GeV}$.

Due to the limited signal statistics, higher thresholds are not applied as they turn out to reduce the signal and decrease the signal significance finally. Events which pass all above cuts fill the di-muon invariant mass distribution. Tables 4 and 5 show the numbers of signal and background events after applying each selection cut at an integrated luminosity of $1000 \mathrm{fb}^{-1}$. The signal total selection efficiencies are within one to three percent. The background events are, however, suppressed to a desirable level.

The resulting di-muon invariant mass distributions are shown in Fig. 10 with $\sqrt{s}=0.5 \mathrm{TeV}$ and Fig. 11 with $\sqrt{s}=1 \mathrm{TeV}$. As seen from Figs. 10 and 11, the signal is observable on top of the background in all benchmark points. The signal peaks are well separated from the $Z$ boson peak. In cases which involve different masses for $H$ and $A$, both Higgs bosons are observable. Therefore this process can be considered as a promising channel for a synchro-

Table 4 Number of signal events after each selection cut. The last row indicates how many events fill the di-muon distribution at $\mathcal{L}=1000 \mathrm{fb}^{-1}$

\begin{tabular}{|c|c|c|c|c|c|c|c|c|}
\hline \multirow[b]{2}{*}{ Benchmark point } & \multicolumn{4}{|c|}{$\sqrt{s}=0.5 \mathrm{TeV}$} & \multicolumn{4}{|c|}{$\sqrt{s}=1 \mathrm{TeV}$} \\
\hline & BP1 & BP2 & BP3 & BP4 & BP5 & BP6 & BP7 & BP8 \\
\hline$\sigma \times \mathcal{L}$ & $2.7 \times 10^{4}$ & $1.9 \times 10^{4}$ & $1.1 \times 10^{4}$ & $4.3 \times 10^{3}$ & $8.8 \times 10^{3}$ & $7.2 \times 10^{3}$ & $6.4 \times 10^{3}$ & $5.4 \times 10^{3}$ \\
\hline 1 jet & $2.2 \times 10^{4}$ & $1.5 \times 10^{4}$ & $9.2 \times 10^{3}$ & $3.5 \times 10^{3}$ & $7.4 \times 10^{3}$ & $6.0 \times 10^{3}$ & $5.3 \times 10^{3}$ & $4.5 \times 10^{3}$ \\
\hline$b$-jet veto & $2.2 \times 10^{4}$ & $1.5 \times 10^{4}$ & $9.2 \times 10^{3}$ & $3.5 \times 10^{3}$ & $7.4 \times 10^{3}$ & $6.0 \times 10^{3}$ & $5.3 \times 10^{3}$ & $4.5 \times 10^{3}$ \\
\hline $1 \tau$ & $2.0 \times 10^{4}$ & $1.4 \times 10^{4}$ & $8.7 \times 10^{3}$ & $3.3 \times 10^{3}$ & $7.1 \times 10^{3}$ & $5.8 \times 10^{3}$ & $5.1 \times 10^{3}$ & $4.4 \times 10^{3}$ \\
\hline$\Delta \phi$ & 489.1 & 352.3 & 232.2 & 96.8 & 316.9 & 262.2 & 235.8 & 202.9 \\
\hline MET & 343.8 & 261.3 & 175.6 & 68.0 & 218.7 & 186.6 & 172.6 & 152.9 \\
\hline $\mathrm{di}-\tau$ & 301.9 & 229.0 & 154.5 & 59.3 & 211.7 & 181.3 & 167.6 & 148.5 \\
\hline Events at $1000 \mathrm{fb}^{-1}$ & 273.2 & 206.8 & 139.3 & 53.0 & 207.1 & 177.2 & 163.4 & 144.6 \\
\hline
\end{tabular}

Table 5 Number of background events after each selection cut. The last row indicates how many events fill the di-muon distribution at $\mathcal{L}=1000 \mathrm{fb}^{-1}$

\begin{tabular}{|c|c|c|c|c|c|c|c|c|}
\hline \multirow[t]{2}{*}{ Background sample } & \multicolumn{4}{|c|}{$\sqrt{s}=0.5 \mathrm{TeV}$} & \multicolumn{4}{|c|}{$\sqrt{s}=1 \mathrm{TeV}$} \\
\hline & WW & $\mathrm{ZZ}$ & $\mathrm{Z}$ & $t \bar{t}$ & WW & $\mathrm{ZZ}$ & $\mathrm{Z}$ & $t \bar{t}$ \\
\hline$\sigma \times \mathcal{L}$ & $7.8 \times 10^{6}$ & $5.8 \times 10^{5}$ & $1.7 \times 10^{7}$ & $5.9 \times 10^{5}$ & $3.2 \times 10^{6}$ & $2.0 \times 10^{5}$ & $4.3 \times 10^{6}$ & $2.1 \times 10^{5}$ \\
\hline 1 jet & $4.2 \times 10^{6}$ & $3.5 \times 10^{5}$ & $4.6 \times 10^{6}$ & $5.8 \times 10^{5}$ & $9.5 \times 10^{5}$ & $7.7 \times 10^{4}$ & $1.1 \times 10^{6}$ & $2.1 \times 10^{5}$ \\
\hline$b$-jet veto & $3.2 \times 10^{6}$ & $2.1 \times 10^{5}$ & $3.1 \times 10^{6}$ & $1.7 \times 10^{4}$ & $7.3 \times 10^{5}$ & $4.5 \times 10^{4}$ & $7.2 \times 10^{5}$ & $8.1 \times 10^{3}$ \\
\hline $1 \tau$ & $1.3 \times 10^{6}$ & $8.3 \times 10^{4}$ & $8.9 \times 10^{5}$ & $1.0 \times 10^{4}$ & $2.0 \times 10^{5}$ & $1.3 \times 10^{4}$ & $1.7 \times 10^{5}$ & $4.3 \times 10^{3}$ \\
\hline$\Delta \phi$ & 1679.1 & 3995.2 & 5849.3 & 230.1 & 834.8 & 850.7 & 2702.6 & 132.2 \\
\hline MET & 359.4 & 3120.7 & 143.7 & 148.7 & 122.3 & 544.5 & 76.5 & 62.4 \\
\hline $\mathrm{di}-\tau$ & 311.6 & 378.5 & 13.3 & 132.6 & 110.7 & 131.7 & 12.8 & 58.0 \\
\hline Events at $1000 \mathrm{fb}^{-1}$ & 208.4 & 269.6 & 10.3 & 85.9 & 82.2 & 110.3 & 10.5 & 40.7 \\
\hline
\end{tabular}



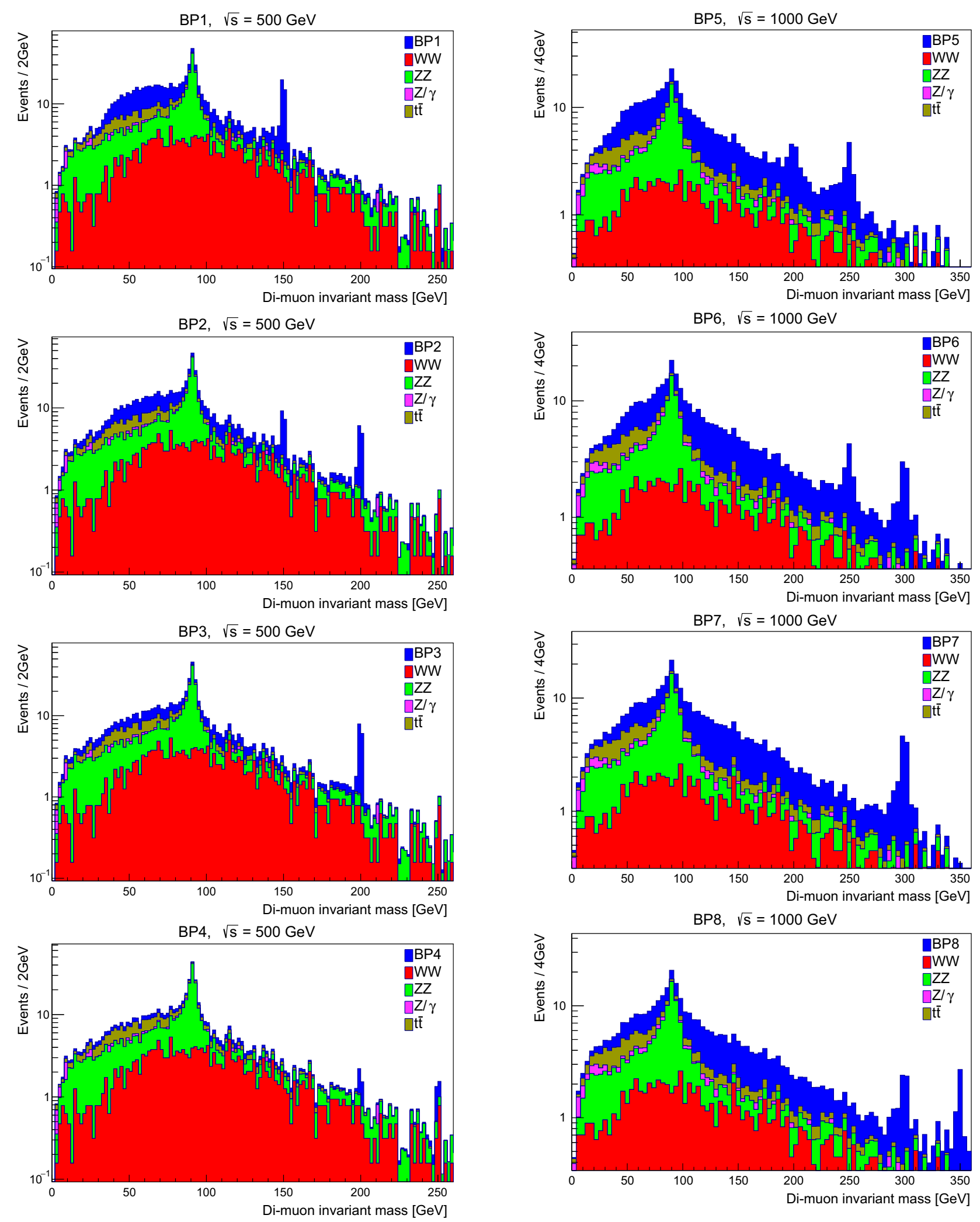

Fig. 10 The di-muon invariant mass in signal and background events at $\sqrt{s}=0.5 \mathrm{TeV}$

Fig. 11 The di-muon invariant mass in signal and background events at $\sqrt{s}=1 \mathrm{TeV}$ 
Table 6 Efficiency of mass window cut applied on signal and background events. The final numbers of events (denoted by $S$ and $B$ ) are obtained as the product of theseefficiencies and the number of events obtained before the mass window cut (the last rows of Tables 4 and 5).The $S / B$ ratio and the signal significance are also shown for an integrated luminosity of $1000 \mathrm{fb}^{-1}$

\begin{tabular}{|c|c|c|c|c|c|c|c|c|c|}
\hline \multirow[t]{2}{*}{ BP } & \multicolumn{5}{|c|}{ Mass window efficiency } & \multicolumn{4}{|c|}{ Final statistics } \\
\hline & Signal & WW & $\mathrm{ZZ}$ & $Z / \gamma^{*}$ & $t \bar{t}$ & $S$ & $B$ & $S / B$ & $S / \sqrt{B}$ \\
\hline \multicolumn{10}{|c|}{$\sqrt{s}=0.5 \mathrm{TeV}$} \\
\hline 1 & 0.122 & $4.5 \times 10^{-2}$ & $1.0 \times 10^{-2}$ & 0.0 & $1.6 \times 10^{-2}$ & 33 & 6.8 & 4.9 & 13 \\
\hline 2 & 0.112 & $3.2 \times 10^{-2}$ & $8.6 \times 10^{-3}$ & 0.0 & $9.2 \times 10^{-3}$ & 23 & 9.8 & 2.3 & 7.4 \\
\hline 3 & 0.092 & $2.0 \times 10^{-2}$ & $6.7 \times 10^{-3}$ & 0.0 & $2.5 \times 10^{-3}$ & 13 & 3 & 4.2 & 7.3 \\
\hline 4 & 0.064 & $1.5 \times 10^{-2}$ & $5.4 \times 10^{-3}$ & 0.0 & $1.4 \times 10^{-3}$ & 3.4 & 4.7 & 0.7 & 1.6 \\
\hline \multicolumn{10}{|c|}{$\sqrt{s}=1 \mathrm{TeV}$} \\
\hline 5 & 0.086 & $4.3 \times 10^{-2}$ & $1.5 \times 10^{-2}$ & $8.2 \times 10^{-3}$ & $2.5 \times 10^{-2}$ & 15 & 6.2 & 2.5 & 6.2 \\
\hline 6 & 0.071 & $2.4 \times 10^{-2}$ & $1.2 \times 10^{-2}$ & $8.2 \times 10^{-3}$ & $1.3 \times 10^{-2}$ & 12 & 4 & 3 & 6 \\
\hline 7 & 0.059 & $1.2 \times 10^{-2}$ & $1.1 \times 10^{-2}$ & $1.6 \times 10^{-2}$ & $9.1 \times 10^{-3}$ & 9.1 & 1.4 & 6.7 & 7.8 \\
\hline 8 & 0.060 & $9.3 \times 10^{-3}$ & $8.1 \times 10^{-3}$ & $8.2 \times 10^{-3}$ & $6.4 \times 10^{-3}$ & 8.3 & 2 & 4.1 & 5.9 \\
\hline
\end{tabular}

nized reconstruction of both scalar and pseudo-scalar Higgs bosons.

In order to provide some figures and statistics, the final quantitative results are outlined in Table 6, which includes the mass window cut efficiencies for signal and background events as well as the final numbers of signal and background denoted by " $\mathrm{S}$ " and " $\mathrm{B}$ " after the mass window cut (at $1000 \mathrm{fb}^{-1}$ ). The mass window for each benchmark point is defined as a $10 \mathrm{GeV}$ wide region centered at the nominal Higgs boson mass. The BP 4 (significance $1.6 \sigma$ at $0.5 \mathrm{TeV}$ ) receives a significance of $6.2 \sigma$ at $1 \mathrm{TeV}$. Therefore all benchmark points are observable at integrated luminosity of $1000 \mathrm{fb}^{-1}$.

\section{Conclusions}

Signals of a neutral Higgs decay to leptons were analyzed at a linear collider operating at $\sqrt{s}=0.5$ and $1 \mathrm{TeV}$. The theoretical framework, i.e., $2 \mathrm{HDM}$ type IV, allows for enhancement of the leptonic decays of $H$ and $A$ bosons. Taking $e^{+} e^{-} \rightarrow A H$ as the signal, two pairs of di-muon and di$\tau$ are produced.

These events are similar in type to SM background processes like $Z Z$. However, a discriminating key feature, i.e., the invariant mass distributions of the two muons from the Higgs boson, can be well separated from the $\mathrm{Z}$ pole mass if $m_{H}>m_{Z}$.

With a detailed analysis of signal and background events, it was shown that the di-muon distribution from signal events can in fact be distinguished from the SM background. The signal to background ratio is large in all cases. The signal significance exceeds $5 \sigma$ in all benchmark points at an integrated luminosity of $1000 \mathrm{fb}^{-1}$.
Acknowledgements I would like to thank Dr. Mogharrab for the operation and maintenance of the computing cluster at Shiraz University, college of sciences.

Open Access This article is distributed under the terms of the Creative Commons Attribution 4.0 International License (http://creativecomm ons.org/licenses/by/4.0/), which permits unrestricted use, distribution, and reproduction in any medium, provided you give appropriate credit to the original author(s) and the source, provide a link to the Creative Commons license, and indicate if changes were made.

Funded by SCOAP S $^{3}$

\section{References}

1. F. Englert, R. Brout, Phys. Rev. Lett. 13, 321 (1964). doi:10.1103/ PhysRevLett.13.321

2. P.W. Higgs, Phys. Rev. Lett. 13, 508 (1964). doi:10.1103/ PhysRevLett.13.508

3. P.W. Higgs, Phys. Lett. 12, 132 (1964). doi:10.1016/ 0031-9163(64)91136-9

4. G.S. Guralnik, C.R. Hagen, T.W.B. Kibble, Phys. Rev. Lett. 13, 585 (1964). doi:10.1103/PhysRevLett.13.585

5. P.W. Higgs, Phys. Rev. 145, 1156 (1966). doi:10.1103/PhysRev. 145.1156

6. T.W.B. Kibble, Phys. Rev. 155, 1554 (1967). doi:10.1103/PhysRev. 155.1554

7. S. Chatrchyan et al., Phys. Lett. B 716, 30 (2012). doi:10.1016/j. physletb.2012.08.021

8. G. Aad et al., Phys. Lett. B 716, 1 (2012). doi:10.1016/j.physletb. 2012.08.020

9. T.D. Lee, Phys. Rev. D 8, 1226 (1973). doi:10.1103/PhysRevD.8. 1226

10. S.L. Glashow, S. Weinberg, Phys. Rev. D 15, 1958 (1977). doi:10 1103/PhysRevD.15.1958

11. G.C. Branco, Phys. Rev. D 22, 2901 (1980). doi:10.1103/ PhysRevD.22.2901

12. I.J.R. Aitchison, Supersymmetry in particle physics. An elementary introduction (Cambridge University Press, Cambridge, UK, 2007), p. 222

13. E. Ma, D. Ng, Phys. Rev. D 49, 6164 (1994). doi:10.1103/ PhysRevD.49.6164 
14. A. Djouadi, Phys. Rept. 459, 1 (2008). doi:10.1016/j.physrep.2007. 10.005

15. J. Mrazek, A. Pomarol, R. Rattazzi, M. Redi, J. Serra, A. Wulzer, Nucl. Phys. B 853, 1 (2011). doi:10.1016/j.nuclphysb.2011.07.008

16. H.E. Haber, D. O'Neil, Phys. Rev. D 74, 015018 (2006). doi:10. 1103/PhysRevD.74.015018, doi:10.1103/PhysRevD.74.059905. [Erratum: Phys. Rev. D 74, no.5,059905(2006)]

17. G.C. Branco, P.M. Ferreira, L. Lavoura, M.N. Rebelo, M. Sher, J.P. Silva, Phys. Rept. 516, 1 (2012). doi:10.1016/j.physrep.2012.02. 002

18. F. Mahmoudi, O. Stal, Phys. Rev. D 81, 035016 (2010). doi:10. 1103/PhysRevD.81.035016

19. S. Kanemura, K. Tsumura, H. Yokoya, Phys. Rev. D 85, 095001 (2012). doi:10.1103/PhysRevD.85.095001

20. S. Davidson, H.E. Haber, Phys. Rev. D 72, 035004 (2005). doi:10. 1103/PhysRevD.72.099902, doi:10.1103/PhysRevD.72.035004. (Erratum: Phys. Rev. D 72,099902(2005))

21. V.D. Barger, J.L. Hewett, R.J.N. Phillips, Phys. Rev. D 41, 3421 (1990). doi:10.1103/PhysRevD.41.3421

22. M. Aoki, S. Kanemura, K. Tsumura, K. Yagyu, Phys. Rev. D 80, 015017 (2009). doi:10.1103/PhysRevD.80.015017

23. G.C. Dorsch, S.J. Huber, K. Mimasu, J.M. No, Phys. Rev. Lett. 113(21), 211802 (2014). doi:10.1103/PhysRevLett.113.211802

24. S. Su, B. Thomas, Phys. Rev. D 79, 095014 (2009). doi:10.1103/ PhysRevD.79.095014

25. W. Grimus, L. Lavoura, O.M. Ogreid, P. Osland, J. Phys. G35, 075001 (2008). doi:10.1088/0954-3899/35/7/075001
26. D. Eriksson, J. Rathsman, O. Stal, Comput. Phys. Commun. 181, 189 (2010). doi:10.1016/j.cpc.2009.09.011

27. D. Eriksson, J. Rathsman, O. Stal, Comput. Phys. Commun. 181, 833 (2010). doi:10.1016/j.cpc.2009.12.016

28. R. Barate et al., Phys. Lett. B 487, 253 (2000). doi:10.1016/ S0370-2693(00)00822-4

29. M. Acciarri et al., Phys. Lett. B 496, 34 (2000). doi:10.1016/ S0370-2693(00)01272-7

30. J. Lee-Franzini, F. Bossi, P. Franzini. in Lepton and photon interactions at high energies. Proceedings, 20th international symposium, LP 2001, Rome, Italy, 23-28 July 2001 (2001)

31. CMS Collaboration, CMS-PAS-HIG-16-037

32. The ATLAS Collaboration, ATLAS-CONF-2016-085

33. M. Misiak et al., Phys. Rev. Lett. 114(22), 221801 (2015). doi:10. 1103/PhysRevLett.114.221801

34. T. Sjostrand, S. Mrenna, P.Z. Skands, Comput. Phys. Commun. 178, 852 (2008). doi:10.1016/j.cpc.2008.01.036

35. M. Cacciari, in Deep inelastic scattering. Proceedings, 14th International Workshop, DIS 2006, Tsukuba, Japan, April 20-24, 2006 (2006), pp. 487-490. (125(2006))

36. M. Cacciari, G.P. Salam, G. Soyez, Eur. Phys. J. C 72, 1896 (2012). doi:10.1140/epjc/s10052-012-1896-2

37. L. Linssen, A. Miyamoto, M. Stanitzki, H. Weerts (2012). doi:10. 5170/CERN-2012-003 